\title{
Prediction of the vibro-acoustic transmission loss of planar hose-pipe systems
}

\author{
M. L. Munjal \\ Department of Mechanical Engineering, Indian Institute of Science, Bangalore 560 012, India \\ P. T. Thawani \\ Climate Control Operations, Ford Motor Company, 15031 S. Commerce Drive, Dearborn, Michigan 48120
}

\begin{abstract}
Vibro-acoustic energy travels through hose walls as longitudinal waves and flexural waves, apart from the sound waves through the fluid medium inside. Longitudinal waves in the hose wall are coupled to the sound waves inside by means of the hose-wall Poisson's ratio. Both in turn get coupled to bending or flexural waves because of the energy transfer or interaction at the bends. For any of these three types of waves incident on one end of a hose, waves of all the three types may be transmitted on the other end because of their dynamical coupling with one another. Therefore, in the present paper, expressions have been derived for the $3 \times 3$ transmission loss matrix for a two-dimensional or planar piping system in terms of elements of the overall $8 \times 8$ transfer matrix of the system. These expressions have then been used in a comprehensive computer program to evaluate the vibro-acoustic performance of hoses, with particular application to the automotive climate control systems with gaseous as well as liquid media. Finally, parametric studies have been made that have led to some general design guidelines. (c) 1997 Acoustical Society of America. [S0001-4966(97)03105-6]
\end{abstract}

PACS numbers: 43.20.Mv, 43.20.Jr, 43.50.Gf [JEG]

\section{INTRODUCTION}

Rubber hoses have been used for several decades for transport of fluids so as to isolate vibrating machinery from accessories like exhaust and intake mufflers or receiver bottles/plenums. Hoses are capable of executing flexural vibrations and torsional vibrations like a beam or shaft. The hose wall can conduct oscillating longitudinal forces that may interact with waves in the fluid inside as well as outside. Flexural forces and moments get coupled to longitudinal forces and acoustic pressures because of bends in the piping system. If the fluid inside were a liquid, its inertia would accentuate the role of the bend.

Wiggert et al. ${ }^{1}$ considered Poisson coupling in a pipe with two bends. Based on linearized assumptions and periodic motion, a simultaneous solution of the coupled fluidstructure system equations in waveform were presented recently by Lesmez et al. ${ }^{2}$ They have derived a composite $14 \times 14$ transfer matrix consisting of submatrices for Poissoncoupled axial stress waves in the wall and pressure waves in the fluid column, flexural waves in the two normal planes, and torsional waves about the axis of the pipe or hose (see Appendix A for the transfer matrix relations and Fig. 1 for the forces and displacements). In their analysis, compliance or yielding of the hose wall is built into a modified bulk modulus for the fluid, and structural damping is accounted for by means of a modified bulk modulus. ${ }^{3,4}$ At higherfrequencies, however, inertia of the wall plays a significant role and must be accounted for by means of wall impedance. The concept of wall impedance, incidentally, enables one to predict transverse transmission loss and thence the breakout noise too. ${ }^{5}$

The concept of transmission loss (TL), which has been used extensively as a measure of the performance of an acoustical filter ${ }^{6}$ or muffler, may also be applied to the structure-borne sound that travels in the form of longitudinal waves and flexural waves. ${ }^{7}$ TL of a one-dimensional acoustical filter has been expressed in terms of the elements of the overall transfer matrix of the filter in the literature (see Ref. 6 for example), but no such expressions are to be found for structure-borne sound. The present paper seeks to fill this gap.

Structure-borne longitudinal waves, flexural waves, and fluid-borne sound waves are dynamically coupled. Hence, a wave of any of these three types when incident on a planar hose-pipe system will in general result in reflection and transmission of all three types of waves. Thus, complete knowledge of the transmission characteristics of a hose system will in general be given by a $3 \times 3$ matrix of transmission loss (TL) values. In this paper, these TL values have been derived in terms of elements of the overall $8 \times 8$ matrix of the hose-pipe system, adapted from Ref. 2 (see Appendix A) wherein the effects of shear deformation, rotary inertia, and the fluid load have been incorporated. These expressions have then been used for parametric studies related to vibroacoustics of hoses. Some general design guidelines based on the parametric studies have been arrived at for visco-elastic hoses, with or without bends, with gases or liquids as the media of propagation.

\section{BASIC GOVERNING EQUATIONS}

Longitudinal waves in the hose wall and onedimensional sound waves in the medium inside are governed by the following coupled equations: 


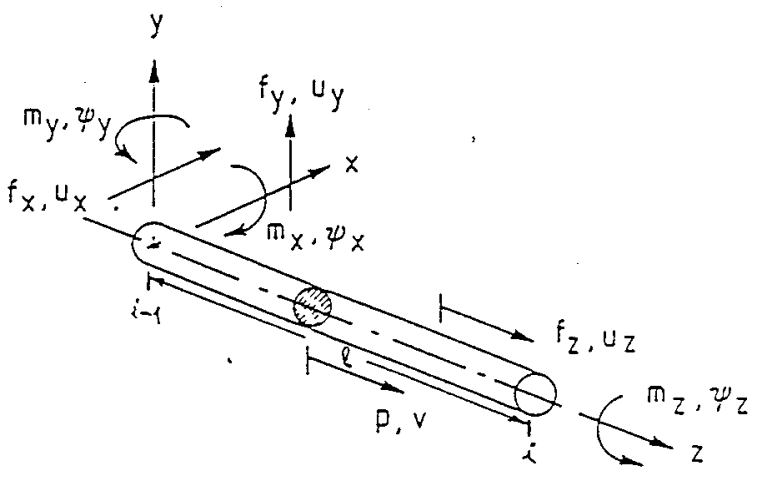

FIG. 1. A straight hose-pipe reach with state variables.

$f_{z}-A_{p} \nu \frac{r}{e} p-E A_{p} \frac{\partial u_{z}}{\partial z}=0$

$\frac{\partial f_{z}}{\partial z}-\rho_{p} A_{p} \frac{\partial^{2} u_{z}}{\partial t^{2}}=0$

$p+K^{*} \frac{\partial v}{\partial z}-2 \nu K^{*} \frac{\partial u_{z}}{\partial z}=0$,

$\frac{\partial p}{\partial z}+\rho_{f} \frac{\partial^{2} v}{\partial t^{2}}=0$

Flexural (or bending) waves are governed by the following equations for a beam incorporating shear deformation, rotary inertia, and fluid inertia:

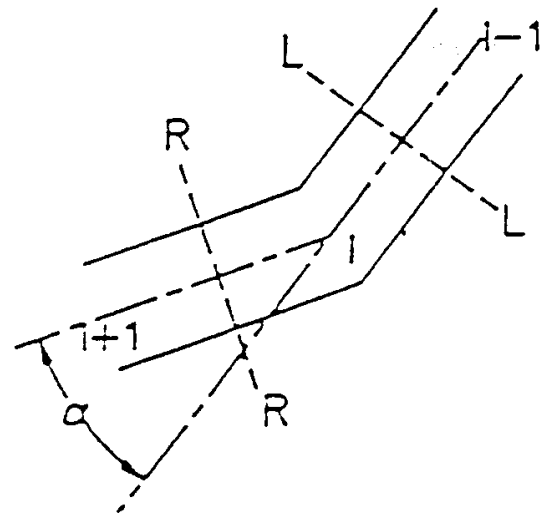

FIG. 2. A sharp bend.

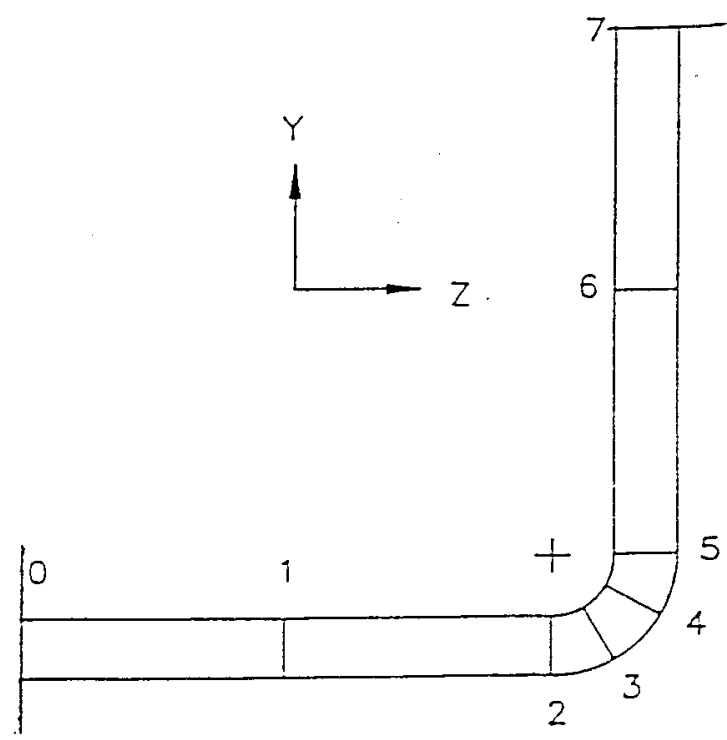

FIG. 3. A hose-pipe with a smooth bend.

shear angle: $\beta_{x}=\frac{\partial u_{y}}{\partial z}+\psi_{x}$,

shear force: $f_{y}=G A_{p} k_{s} \beta_{x}$,

bending moment: $m_{x}=E I_{p} \frac{\partial \psi_{x}}{\partial z}$,

force equilibrium: $\frac{\partial f_{y}}{\partial z}-\left(\rho_{p} A_{p}+\rho_{f} A_{f}\right) \frac{\partial^{2} u_{y}}{\partial t^{2}}=0$,

moment equilibrium: $\frac{\partial m_{x}}{\partial z}-f_{y}-\left(\rho_{p} I_{p}+\rho_{f} I_{f}\right) \frac{\partial^{2} \psi}{\partial t^{2}}=0$,

where $f_{z}, u_{z}, p, v, m_{x}, \psi_{x}, f_{y}$, and $u_{y}$ are state variables as shown in Fig. 1. Other notations are as follows: $A_{p}$ is the cross-sectional area of the pipe, $\nu$ is the Poisson's ratio of the pipe material, $r$ is radius of the pipe cross section, $e$ is the pipe wall thickness, $E$ is Young's modulus (generally complex), $z$ is the axial coordinate as shown in Fig. $1, \rho_{p}$ is density of the pipe material, $\rho_{f}$ is density of the fluid inside the pipe, $G$ is the shear modulus of the pipe material, $k_{s}$ is the shear shape factor, $I_{p}$ is the moment of inertia of the pipe, $I_{f}$ is the moment of inertia of the fluid inside the pipe, $K^{*}$ is the modified bulk modulus; for thin-walled pipes,

$$
K^{*}=\frac{K}{1+K\left(1-\nu^{2}\right) r / E e},
$$

where $K$ is the bulk modulus of the fluid.

\section{EVALUATION OF THE TL MATRIX}

For a one-dimensional hose-line system configured in the $y-z$ plane (the plane of the paper), field matrices (A6) for straight pipes (Fig. 1) and point matrices (A8) of Appendix A for sharp bands (Fig. 2) may be multiplied successively as per configuration so as to obtain the overall or product transfer matrix relation connecting the state vector ${ }^{2}$ 


$$
\left\{\begin{array}{llllllll}
U_{z} & P & V & F_{z} & U_{y} & \Psi_{x} & M_{x} & F_{y}
\end{array}\right\}^{T}
$$

on the right-hand (or downstream) side with that on the left hand (or upstream) side (see Fig. 1). Here $U, P, V, F, \Psi$, and $M$ denote complex amplitudes of displacement, acoustic pressure, acoustic particle displacement, force, flexural angular rotation (or slope), and bending moment, respectively. Field matrix of the constituent hose-pipe elements would be made up of the $4 \times 4$ submatrices $T_{f p}$ and $T_{y z}$ only. Before multiplying out, all transfer matrix elements have to be multiplied with the dimensional factors associated with the state variables (A7), so that state variables have their physical dimensions as in the state vector (11). It may be noted that for a smooth bend (Fig. 3) the bent portion will have to be partitioned and the angular changes will be lumped at sections $2,3,4$, and $5\left(15^{\circ}, 30^{\circ}, 30^{\circ}, 15^{\circ}\right)$ and the intermediate portions will be treated as curved pipes with modified flexural rigidity $E I .^{2}$

In the absence of any bends, the resultant transfer matrix would consist of two uncoupled $4 \times 4$ submatrices, one representing the product of the individual $T_{f p}$ matrices and the other of the $T_{y z}$ matrices. Further, if we put Poisson's ratio $\nu$ hypothetically equal to zero in the $T_{f p}$ matrix (that is, if we neglect Poisson coupling), then it would separate into two uncoupled $2 \times 2$ submatrices corresponding to longitudinal waves in the hose wall and acoustic waves in the fluid medium inside the hose. Thus,

$$
\begin{aligned}
& {\left[\begin{array}{l}
U_{z} \\
F_{z}
\end{array}\right]_{d}=\left[\begin{array}{ll}
T_{11} & T_{14} \\
T_{41} & T_{44}
\end{array}\right]\left[\begin{array}{l}
U_{z} \\
F_{z}
\end{array}\right]_{u}} \\
& {\left[\begin{array}{l}
P \\
V
\end{array}\right]_{d}=\left[\begin{array}{ll}
T_{22} & T_{23} \\
T_{32} & T_{33}
\end{array}\right]\left[\begin{array}{l}
P \\
V
\end{array}\right]_{u}}
\end{aligned}
$$

and

$$
\left[\begin{array}{c}
U_{y} \\
\Psi_{x} \\
M_{x} \\
F_{y}
\end{array}\right]_{d}=\left[\begin{array}{llll}
T_{55} & T_{56} & T_{57} & T_{58} \\
T_{65} & T_{66} & T_{67} & T_{68} \\
T_{75} & T_{76} & T_{77} & T_{78} \\
T_{85} & T_{86} & T_{87} & T_{88}
\end{array}\right]\left[\begin{array}{c}
U_{y} \\
\Psi_{x} \\
M_{x} \\
F_{y}
\end{array}\right]_{u},
$$

where $[T]$ is the overall transfer matrix, and subscripts $d$ and $u$ denote downstream and upstream variables, respectively.

In general, and particularly in the presence of bends, one type of input (incident wave) will result in an output (transmitted wave) of not only the same kind, but also of other kinds. So, one should try to predict a set of nine TLs corresponding to three types of incident waves and three types of transmitted waves, making use of the entire $8 \times 8$ transfer matrix of a planar hose-pipe system [see Eqs. (46)-(52)].

As TL is a symmetric function for stationary medium, state vectors subscripted $u$ and $d$ may be interchanged without having to invert the overall transfer matrix $[T]$. Thus,

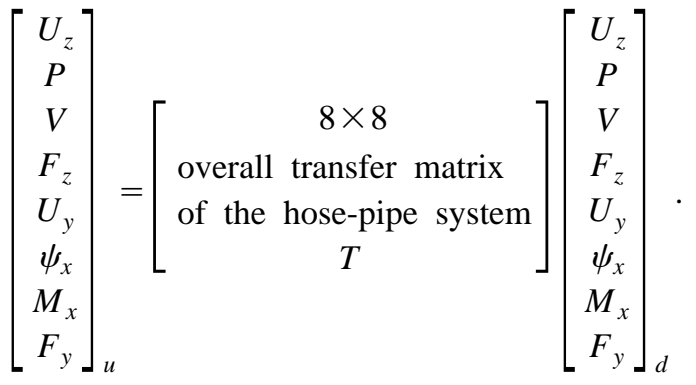

For an incoming or incident progressive wave, there will be one reflected wave and one transmitted wave in the case of sound waves and longitudinal waves (in the hose-pipe wall), and two reflected waves and two transmitted waves, one propagating and one evanescent (exponentially decaying) in the case of flexural waves. Thus, in general, there will be four reflected waves and four transmitted waves (anechoic termination is presumed on the downstream side as implied in the definition of transmission loss). Substituting these into the state variables in Eq. (15) would yield a set of eight inhomogeneous equations. These may be solved to obtain the three propagating type progressive waves (evanescent flexural wave carries no wave energy) in terms of the incident wave, and thence the corresponding three transmission loss values corresponding to this particular type of incident wave. The process can be repeated for the other two types of incident waves to obtain all nine elements of the desired $3 \times 3 \mathrm{TL}$ matrix.

In the following analysis, the (known) incident wave amplitude is denoted by $A$ and the (unknown) reflected and transmitted wave amplitudes (complex in general) by $B$.

Substituting

$$
\left(u_{z}, f_{z}, p, v\right)=\left(U_{z}, F_{z}, P, V\right) e^{\lambda z / l} e^{j \omega t}
$$

into the homogeneous set of Eqs. (1)-(4), and applying the compatibility criterion, yields the characteristic equation

$$
\lambda^{4}+(\tau+\sigma+\gamma) \lambda^{2}+\sigma \tau=0,
$$

where $\tau, \sigma$, and $\gamma$ are defined by the identities (A1a) to (A1c) of Appendix A. Equation (17) has roots

$$
\lambda= \pm j \lambda_{1}, \pm j \lambda_{2},
$$

where $\lambda_{1}$ and $\lambda_{2}$ are given by Eqs. (A11) and (A1m), and represent wave numbers for the longitudinal wave and sound wave, respectively. Therefore, for prediction of transmission loss of longitudinal waves and sound waves, the standing wave solutions may be written as

$$
\begin{aligned}
& u_{z}=\left\{A_{1} e^{-j \lambda_{1} z / l}+B_{1} e^{+j \lambda_{1} z / l}\right\} e^{j \omega t}, \\
& F_{z}=\left\{Q\left(-j \lambda_{1}\right) A_{1} e^{-j \lambda_{1} z / l}+Q\left(j \lambda_{1}\right) B_{1} e^{j \lambda_{1} z / l}\right\} e^{j \omega t}, \\
& v=\left\{A_{2} e^{-j \lambda_{2} z / l}+B_{2} e^{j \lambda_{2} z / l}\right\} e^{j \omega t}, \\
& p=\left\{H\left(-j \lambda_{2}\right) A_{2} e^{-j \lambda_{2} z / l}+H\left(j \lambda_{2}\right) B_{2} e^{j \lambda_{2} z / l}\right\} e^{j \omega t},
\end{aligned}
$$

where functions $Q(\beta)$ and $H(\beta)$ are given by Eqs. (2) and (4) as

$$
Q(\beta)=-\frac{\rho_{p} A_{p} \omega^{2}}{\beta}, \quad \beta \equiv \frac{d}{d z}=\mp j \lambda_{1} / l=\mp j k_{1},
$$




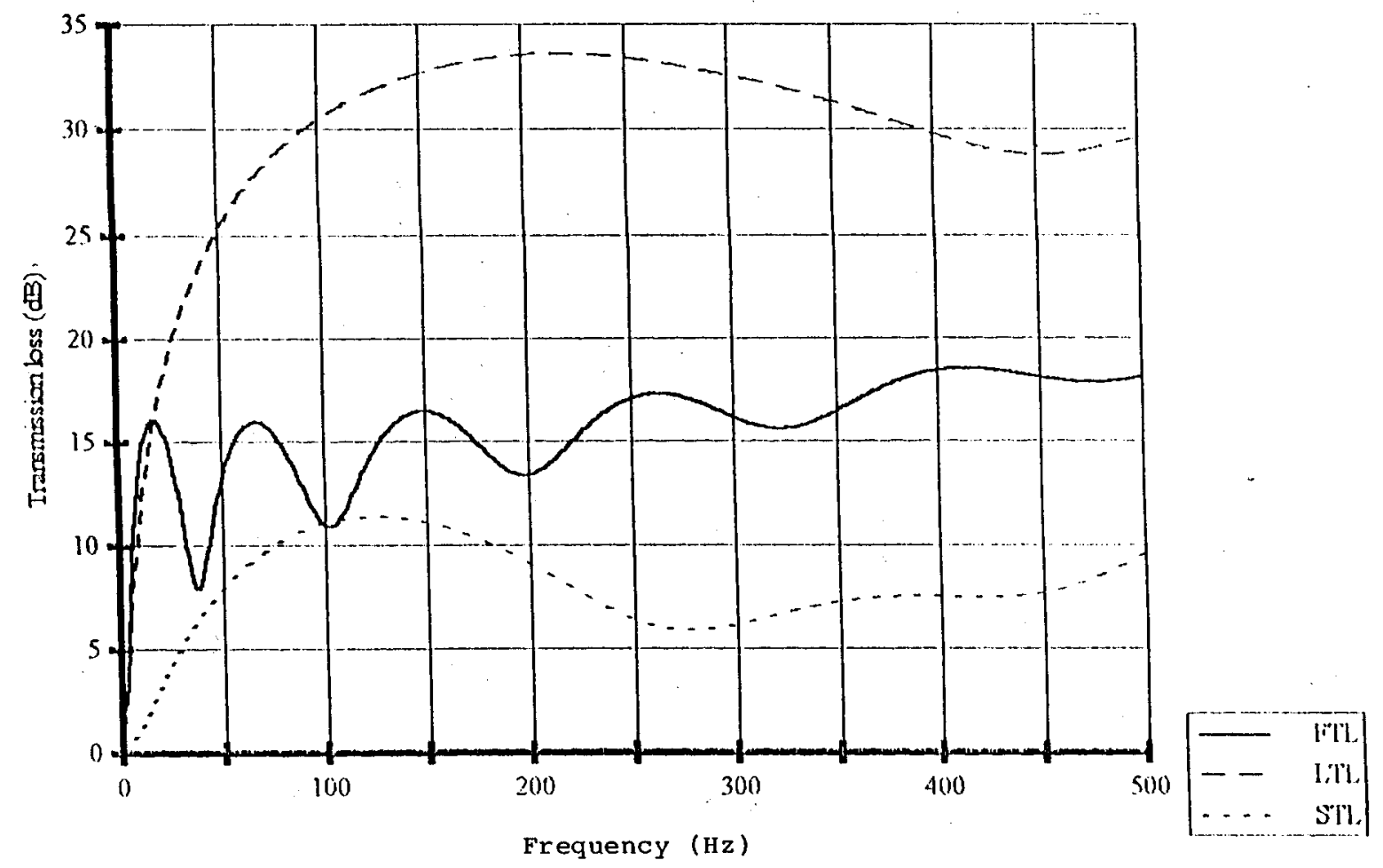

FIG. 4. Vibro-acoustic isolation of a composite rubber hose with liquid medium.

$$
H(\beta)=\frac{\rho_{f} \omega^{2}}{\beta}, \quad \beta \equiv \frac{d}{d z}=\mp j \lambda_{2} / l=\mp j k_{2} .
$$

Putting $z=0$ arbitrarily at the upstream as well as downstream end of the hose-pipe system, and assuming the downstream termination to be anechoic as required by definition of TL, one gets

$$
\begin{aligned}
& U_{z, u}=A_{1}+B_{1}, \quad U_{z, d}=B_{2}, \\
& V_{u}=A_{2}+B_{3}, \quad V_{d}=B_{4}, \\
& P_{u}=H\left(-j k_{u, 2}\right) A_{2}+H\left(j k_{u, 2}\right) B_{3}, \quad p_{d}=B_{4} H\left(-j k_{d, 2}\right),
\end{aligned}
$$

$$
F_{z, u}=Q\left(-j k_{u, 1}\right) A_{1}+Q\left(j k_{u, 1}\right) B_{1},
$$$$
F_{z, d}=B_{2} Q\left(-j k_{d, 1}\right) \text {. }
$$

The corresponding relations for flexural waves may be obtained as follows.

Substituting

$$
\left(u_{y}, \psi_{x}, m_{x}, f_{y}\right)=\left(U_{y}, \Psi_{x}, M_{x}, F_{y}\right) e^{\lambda z / l} e^{j \omega t}
$$

into Eqs. (5)-(9), eliminating shear angle $\beta_{x}$, and applying the compatibility criterion, yields the characteristic equation

$$
\lambda^{4}+(\sigma+\tau) \lambda^{2}-(\gamma-\sigma \tau)=0,
$$

where $\tau, \sigma$, and $\gamma$ are defined by identities (A3a) to (A3c) of Appendix A. Equation (28) has roots

$$
\lambda= \pm \lambda_{3}, \pm j \lambda_{4},
$$

where $\lambda_{3}$ and $\lambda_{4}$ are given by Eqs. (A3i) and (A3j) of Appendix $\mathrm{A}$, and represent evanescent waves and propagating waves, respectively. Therefore, for prediction of flexural transmission loss, the general solutions may be written in terms of $\lambda_{3}$ and $j \lambda_{4}$ as a sum of four progressive waves (two evanescent and two propagating) similar to Eqs. (19) and (20).

Putting $z=0$ arbitrarily at the upstream as well as downstream end of the hose-pipe system, and assuming the downstream termination to be anechoic as required by definition of TL, yields

$$
\begin{aligned}
& U_{y, u}=A_{3}+B_{5}+B_{6}, \quad U_{y, d}=B_{7}+B_{8}, \\
& \Psi_{x, u}=E\left(-j k_{u, 4}\right) A_{3}+E\left(j k_{u, 4}\right) B_{5}+E\left(k_{u, 3}\right) B_{6}, \\
& \Psi_{x, d}=E\left(-j k_{d, 4}\right) B_{7}+E\left(-k_{d, 3}\right) B_{8}, \\
& M_{x, u}=F\left(-j k_{u, 4}\right) A_{3}+F\left(j k_{u, 4}\right) B_{5}+F\left(k_{u, 3}\right) B_{6}, \\
& M_{x, d}=F\left(-j k_{d, 4}\right) B_{7}+F\left(-k_{d, 3}\right) B_{8}, \\
& F_{y, u}=G\left(-j k_{u, 4}\right) A_{3}+G\left(j k_{u, 4}\right) B_{5}+G\left(k_{u, 3}\right) B_{6}, \\
& F_{y, d}=G\left(-j k_{d, 4}\right) B_{7}+E\left(-k_{d, 3}\right) B_{8},
\end{aligned}
$$

where $E(\beta), F(\beta)$, and $G(\beta)$ are calculated from Eqs. (5)(9) as

$$
\begin{aligned}
& E(\beta)=\frac{-\omega^{2} C / \beta}{E I \beta^{2}+D \omega^{2}}, \\
& F(\beta)=-\frac{E I \omega^{2} / C}{E I \beta^{2}+D \omega^{2}}, \\
& G(\beta)=-C \omega^{2} / \beta, \\
& \beta \equiv \frac{d}{d z}=\mp \lambda_{3} / l, \quad \mp j \lambda_{4} / l=\mp k_{3}, \mp j k_{4},
\end{aligned}
$$




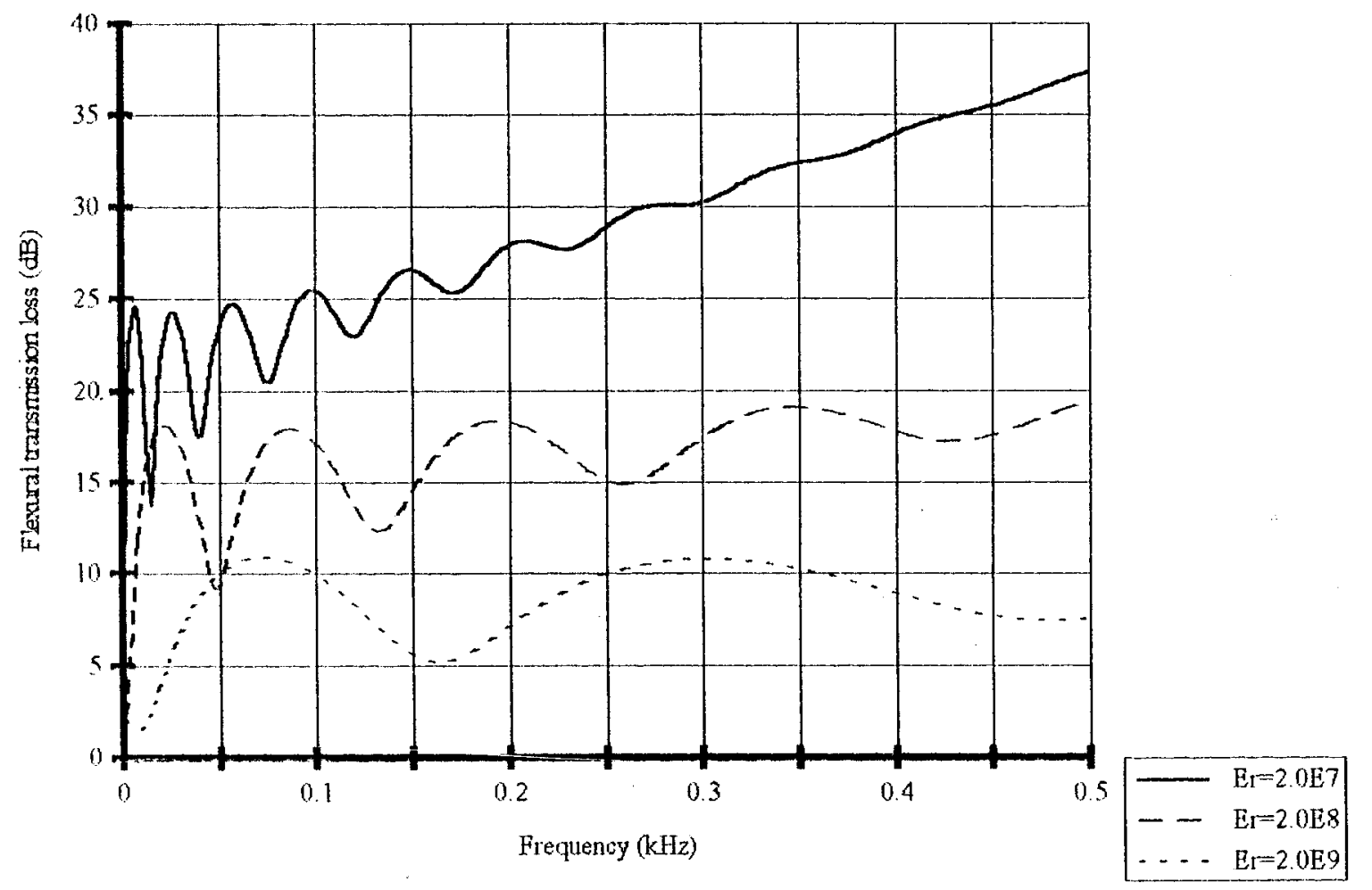

FIG. 5. Effect of the wall storage modulus of a hose on its vibration isolation.

$$
\begin{aligned}
& C \equiv \rho_{p} A_{p}+\rho_{f} A_{f}, \\
& D \equiv \rho_{p} I_{p}+\rho_{f} I_{f} .
\end{aligned}
$$

Substituting Eqs. (25)-(28) and (32)-(35) into the transfer matrix equations (15), and rearranging, yields a set of eight inhomogeneous equations for the eight unknowns $B_{1}$ to $B_{8}$ :

$$
[A]\{B\}=\{S\},
$$

where

$$
\begin{aligned}
& \{B\}=\left[\begin{array}{llllllll}
B_{1} & B_{2} & B_{3} & B_{4} & B_{5} & B_{6} & B_{7} & B_{8}
\end{array}\right]^{T}, \\
& A_{11}=-1, \quad A_{23}=-H\left(j k_{u, 2}\right), \quad A_{33}=-1 \text {, } \\
& A_{41}=-Q\left(j k_{u, 1}\right) \text {, } \\
& A_{55}=-1, \quad A_{56}=-1, \quad A_{65}=-E\left(j k_{u, 4}\right), \\
& A_{66}=-E\left(k_{u, 3}\right), \\
& A_{75}=-F\left(j k_{u, 4}\right), \quad A_{76}=-F\left(k_{u, 3}\right), \\
& A_{85}=-G\left(k_{u, 4}\right), \quad A_{86}=-G\left(k_{u, 3}\right), \\
& A_{i 2}=T_{i 1}+Q\left(-j k_{d, 1}\right) T_{i 4}, \quad i=1,2, \ldots, 8, \\
& A_{i 4}=T_{i 2}+Q\left(-j k_{d, 2}\right) T_{i 3}, \quad i=1,2, \ldots, 8, \\
& A_{i 7}=T_{i 5}+E\left(-j k_{d, 4}\right) T_{i 6}+F\left(-j k_{d, 4}\right) T_{i 7} \\
& +G\left(-j k_{d, 4}\right) T_{i 8}, \quad i=1,2, \ldots, 8 .
\end{aligned}
$$

The rest of the elements of matrix $[A]$ are equal to zero:

$$
\begin{aligned}
& S_{1}=A_{1}, \quad S_{2}=H\left(-j k_{u, 2}\right) A_{2}, \quad S_{3}=A_{2}, \\
& S_{4}=Q\left(-j k_{u, 1}\right) A_{1}, \\
& S_{5}=a_{3}, \quad S_{6}=H\left(-j k_{u, 4}\right) A_{3}, \\
& S_{7}=F\left(-j k_{u, 4}\right) A_{3}, \quad S_{8}=G\left(-j k_{u, 4}\right) A_{3} .
\end{aligned}
$$

The matrix equations (42) are solved by means of a standard subroutine for vector $\{B\}$ for given (say unity) values of the incident waves $A_{1}, A_{2}$, and $A_{3}$ representing longitudinal, acoustic, and flexural waves, respectively.

Now, the expressions for power flux associated with different types of waves can be given by longitudinal waves:

$$
\begin{aligned}
& \text { longitudinal waves: } \quad W_{1}=\frac{\omega}{2} \operatorname{Re}\left[F_{z} \cdot U_{z}^{*}\right] \\
& \text { sound waves: } W_{S}=\frac{\omega}{2} A_{f} \operatorname{Re}\left[P \cdot V^{*}\right] \\
& \text { flexural waves: } W_{f}=\frac{\omega}{4} \operatorname{Re}\left[F_{y} \cdot U_{y}^{*}+M_{x} \cdot \Psi_{x}^{*}\right],
\end{aligned}
$$

where the superscript asterisk indicates complex conjugate.

Applying these expressions to incident waves and transmitted (propagating) waves of the three types, yields

$$
\begin{aligned}
& W_{1, i} \equiv W_{l, i}=\frac{\omega}{2} \operatorname{Re}\left[Q\left(-j k_{u, 1}\right)\right]\left|A_{1}\right|^{2}, \\
& W_{1, t} \equiv W_{l, t}=\frac{\omega}{2} \operatorname{Re}\left[Q\left(-j k_{d, 1}\right)\right]\left|B_{2}\right|^{2},
\end{aligned}
$$




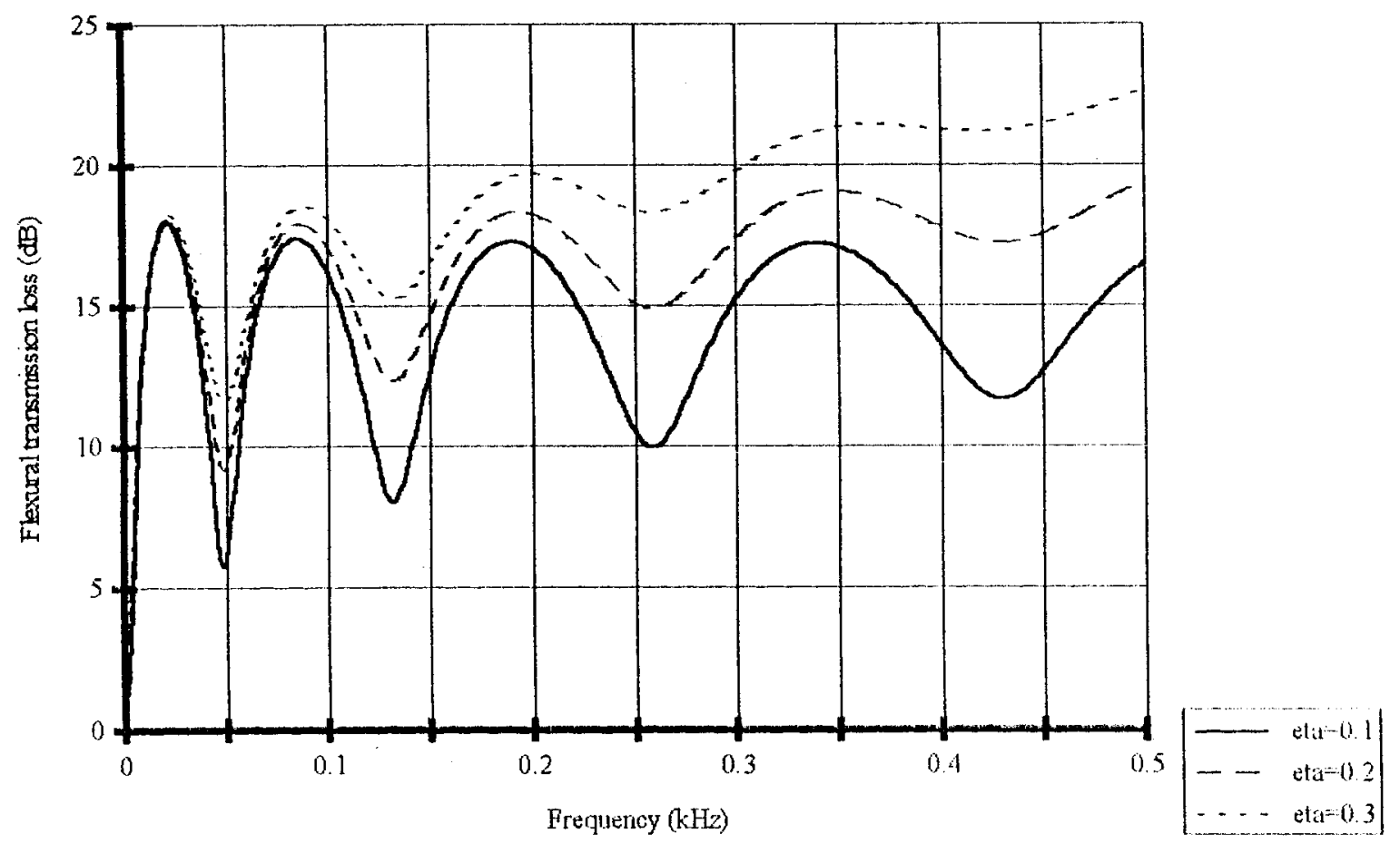

FIG. 6. Effect of the structural damping (loss factor) of a hose on its vibration isolation.

$$
\begin{aligned}
W_{2, i} \equiv W_{s, i}= & \frac{\omega}{2} A_{f, u} \operatorname{Re}\left[H\left(-j k_{u, 2}\right)\right]\left|A_{4}\right|^{2}, \\
W_{2, t} \equiv W_{s, t}= & \frac{\omega}{2} A_{f, d} \operatorname{Re}\left[H\left(-j k_{d, 2}\right)\right]\left|B_{4}\right|^{2}, \\
W_{3, i} \equiv W_{f, i}= & \frac{\omega}{4} \operatorname{Re}\left[G\left(-j k_{u, 4}\right)+F\left(-j k_{u, 4}\right)\right. \\
& \left.\times E^{*}\left(-j k_{u, 4}\right)\right]\left|A_{3}\right|^{2}, \\
W_{3, t} \equiv W_{f, t}= & \frac{\omega}{4}\left[G\left(-j k_{d, 4}\right)+F\left(-j k_{d, 4}\right)\right. \\
& \left.\times E^{*}\left(-j k_{d, 4}\right)\right]\left|B_{7}\right|^{2} .
\end{aligned}
$$

Finally, elements of the $3 \times 3$ matrix of transmission loss may be calculated by means of the equation:

$$
\begin{aligned}
\operatorname{TLM}(j 1, j 2)= & 10 \log \frac{W_{j 1, i}}{W_{j 2, t}}(\mathrm{~dB}), \\
& j 1, j 2=1,2 \text {, and } 3 .
\end{aligned}
$$

For straight hose-pipe systems, there is no coupling between flexural waves and the other two waves. Therefore, for systems without any bends, $\operatorname{TLM}(1,3)=\operatorname{TLM}(2,3)=\operatorname{TLM}(3,1)$ $=\operatorname{TLM}(3,2)=0$. Similarly, if one neglects Poisson coupling between longitudinal waves in the hose wall and the sound waves in the fluid inside, then $\operatorname{TLM}(1,2)=\operatorname{TLM}(2,1)=0$. For most practical applications, only the diagonal elements of the TL matrix are of relevance. These are
$\operatorname{TLM}(1,1)=\mathrm{TL}_{l} \equiv \mathrm{LTL}$

(TL of longitudinal waves in the hose wall),

$\operatorname{TLM}(2,2)=\mathrm{TL}_{s} \equiv \mathrm{STL}$

(TL of longitudinal waves in the fluid inside),

$\operatorname{TLM}(3,3)=\mathrm{TL}_{f} \equiv \mathrm{FTL}$ (TL of flexural waves).

In the presence of bends, however, coupling terms of the TL matrix may also become relevant because of cross-mode energy transfer.

\section{TYPICAL RESULTS AND CONCLUSIONS}

A comprehensive computer program has been prepared in FORTRAN for prediction of the transmission loss values for longitudinal waves, sound waves, and flexural waves (LTL, STL, and FTL), making use of the expressions derived above. Parametric studies were made for different materials and geometries as listed in Appendix B, for flexural waves ( $\mathrm{TL}_{f}$ or FTL).

Typical results are shown in Fig. 4 for the default configuration of a 500-mm long, 19-mm nominal diameter, composite-rubber hose with steel-pipe terminations, but with a liquid medium (power-steering system oil: see Appendix B for specifications). Dips in the TL curves correspond to resonance frequencies that roughly correspond to $k l=n \pi$, $n=1,2,3, \ldots$, where

$$
k=\frac{\omega}{\left(K^{*} / \rho_{f}\right)^{1 / 2}} \text { for sound waves, }
$$




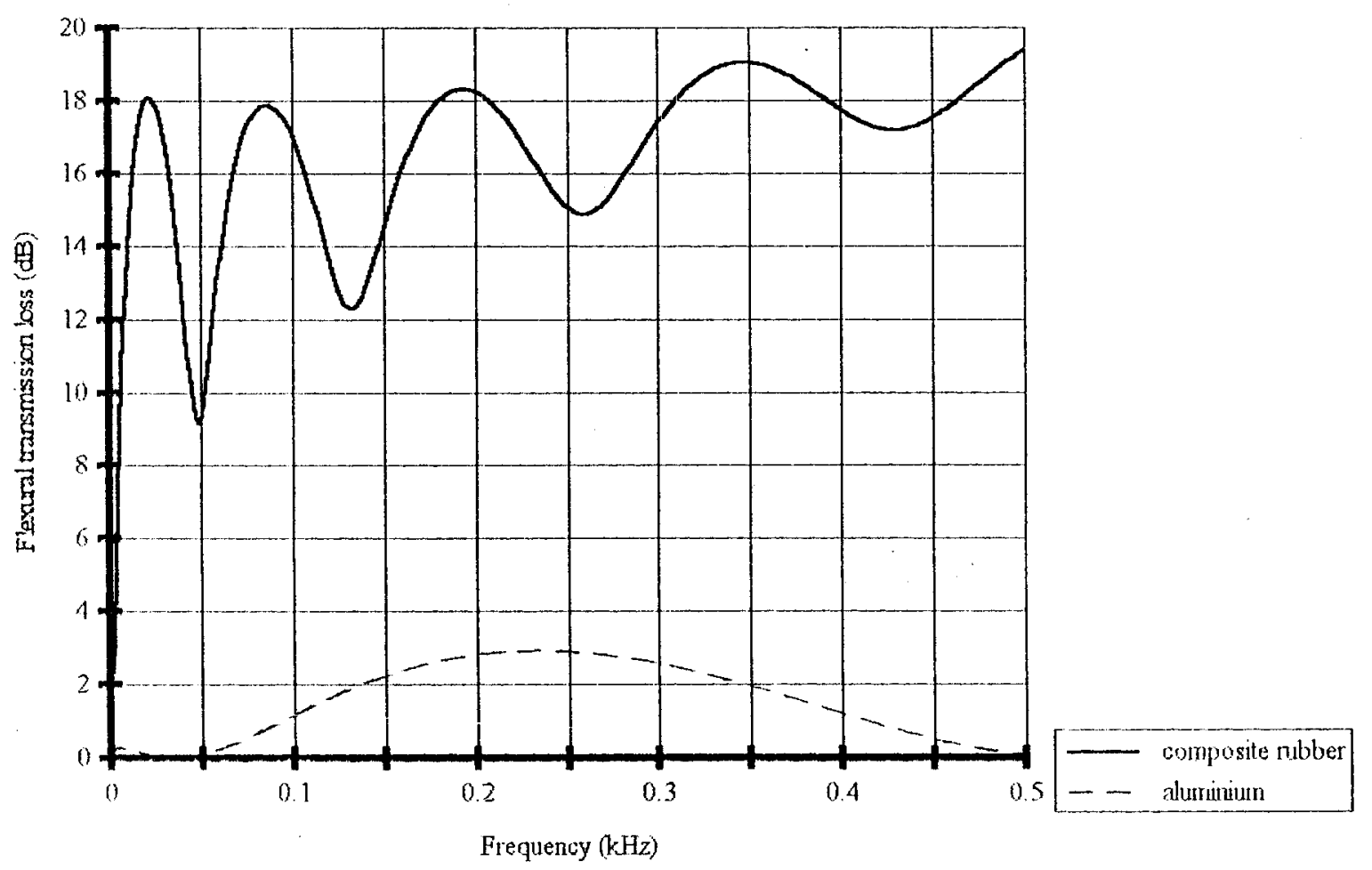

FIG. 7. Effect of the material of a hose on its vibration isolation.
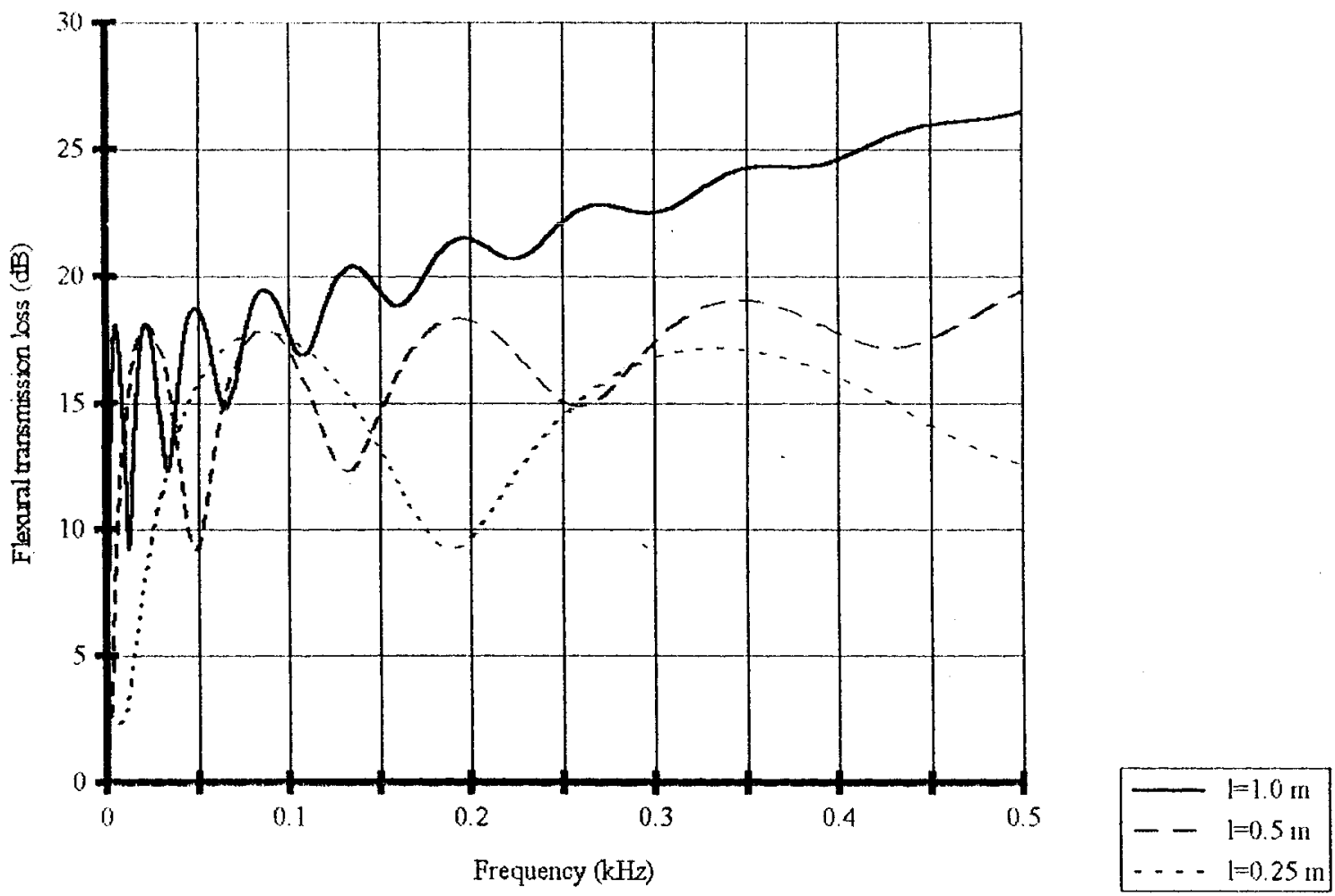

FIG. 8. Effect of the length of a hose on its vibration isolation. 


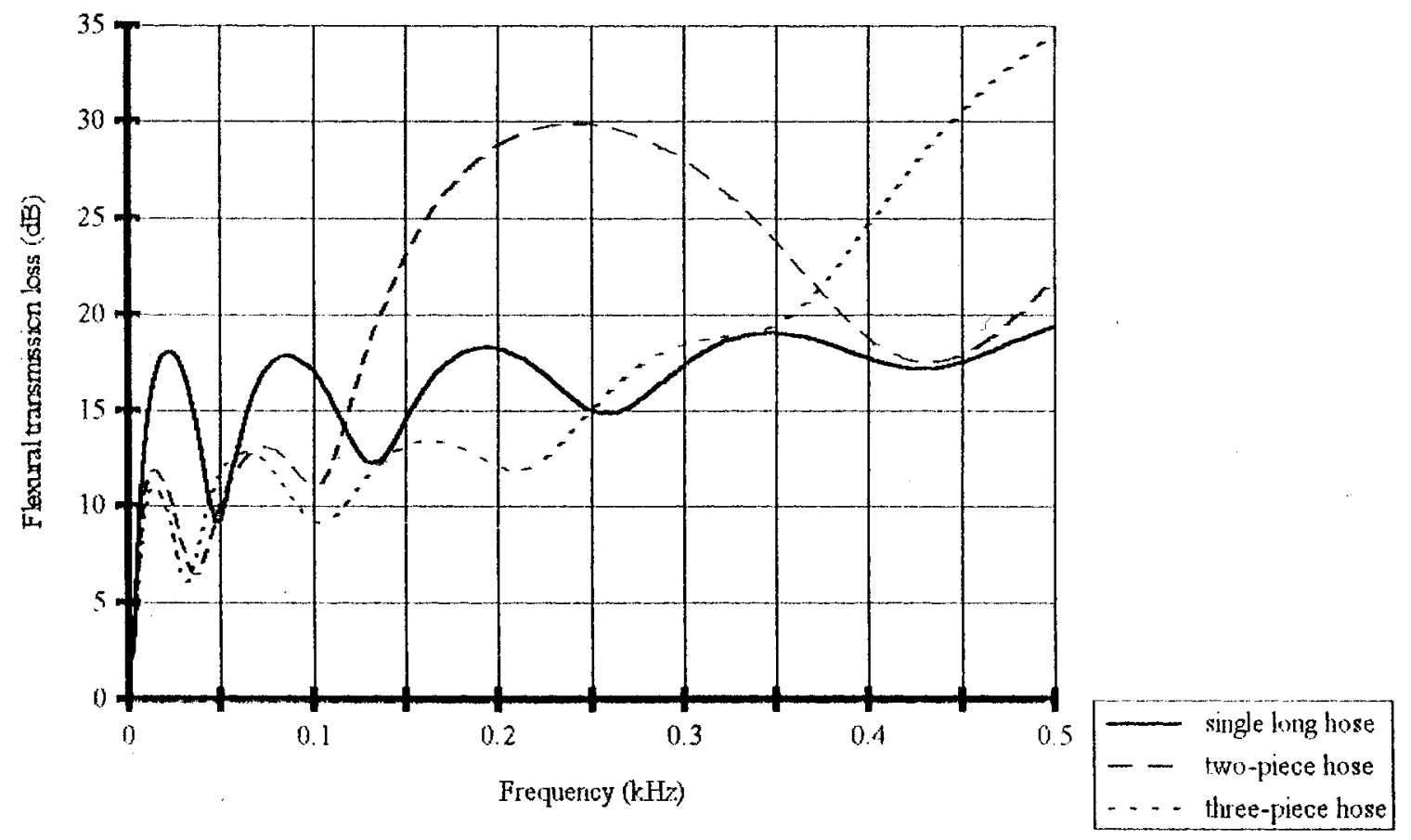

FIG. 9. Effect of the number of hose pieces within the same overall length on vibration isolation.

and

$$
k=\frac{\omega}{\left(E_{r} / \rho_{p}\right)^{1 / 2}} \text { for longitudinal waves. }
$$

The observed values are within $20 \%$ of those given by the approximate relationships (56) and (57).
However, this simple relation $(k l=n \pi)$ does not hold for flexural waves for which

$$
k=\left(\frac{\rho_{p} A_{p} \omega^{2}}{E_{r} I_{p}}\right)^{1 / 4} .
$$

If the medium were a gas, LTL and FTL curves would re-
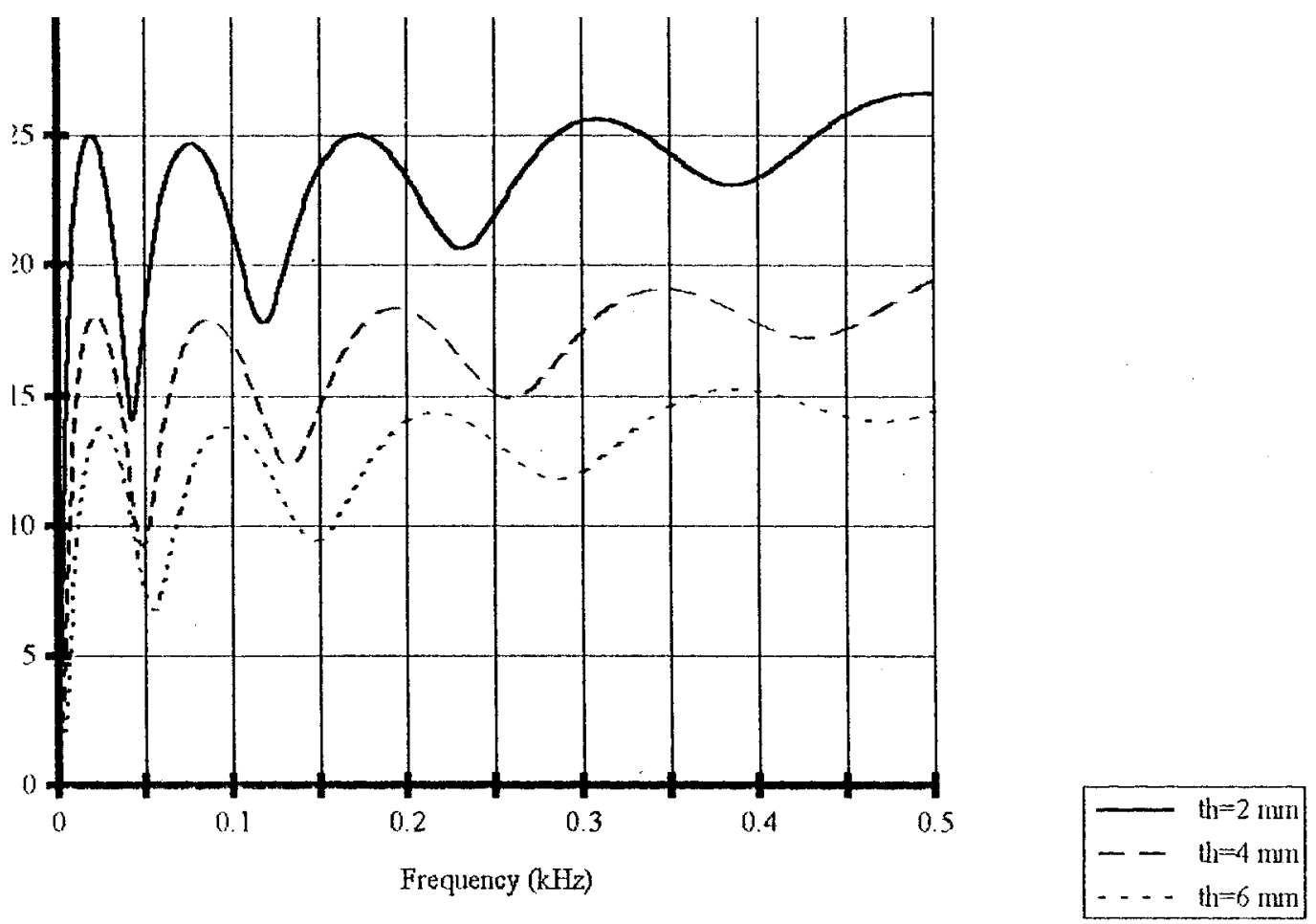

FIG. 10. Effect of wall thickness of a hose on its vibration isolation. 


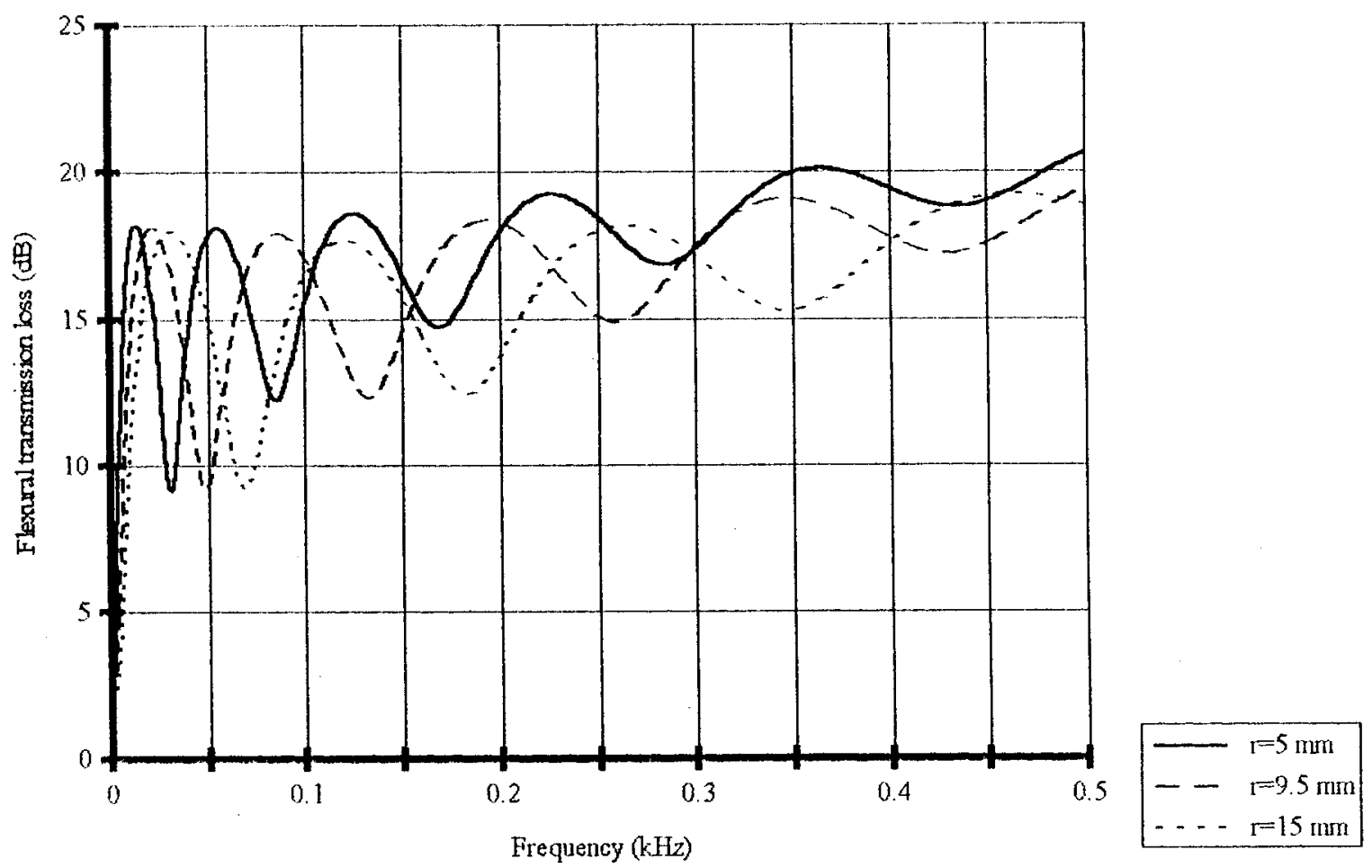

FIG. 11. Effect of the inner radius of a hose on its vibration isolation.

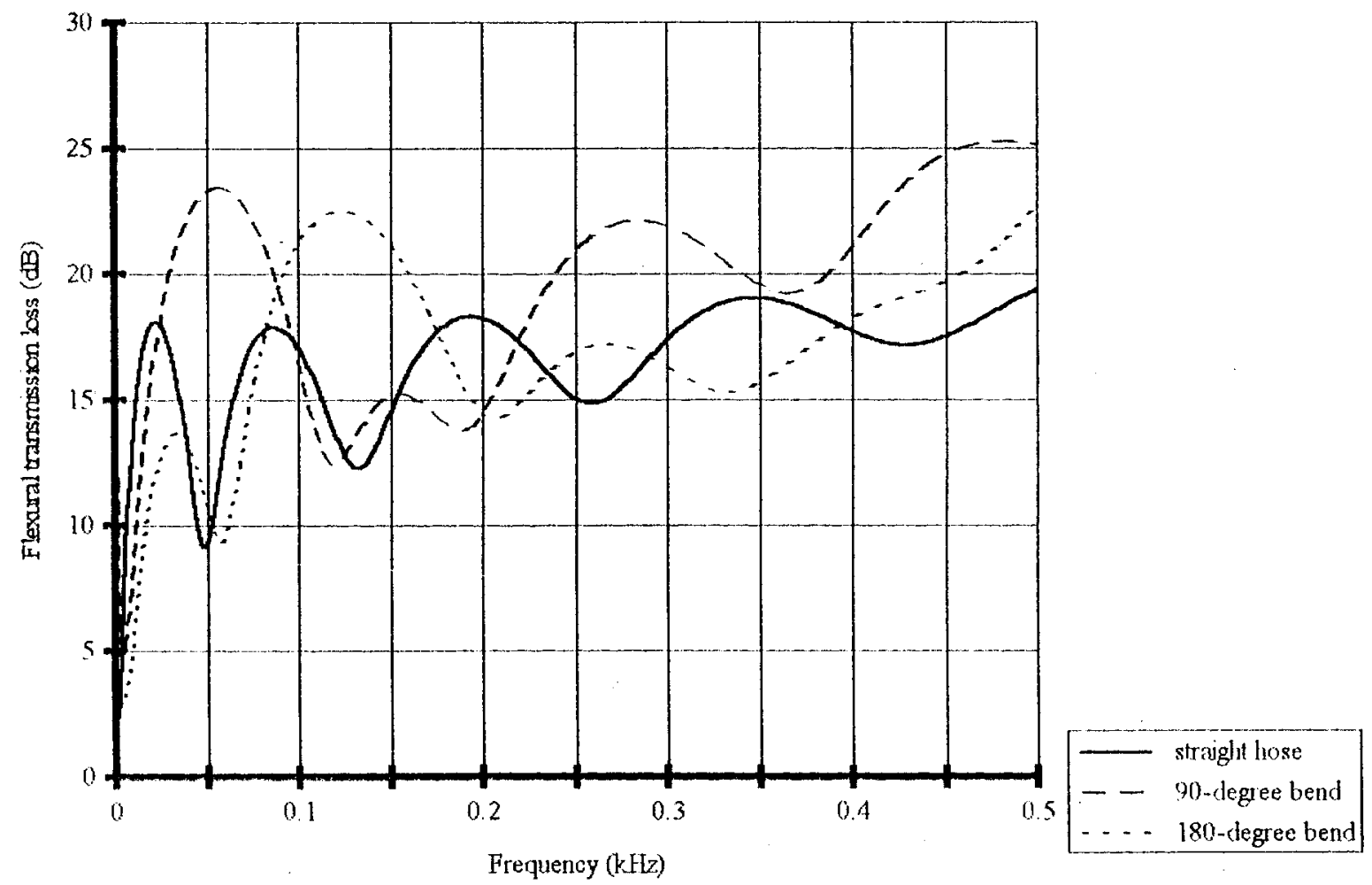

FIG. 12. Effect of bend angle on vibration isolation of a hose. 
main more or less unchanged, but STL would reduce to zero (less than $0.5 \mathrm{~dB}$ ). This is because longitudinal and flexural waves travel in the wall material whereas sound waves travel in the fluid medium inside. For air medium, impedance mismatch between air and hose material is so strong that the wall behaves more or less like a rigid boundary. For liquid medium, however, the impedance mismatch is rather weak.

Poisson coupling is not of much significance because its neglect (putting $\nu=0$ in the $T_{f p}$ transfer matrix) alters LTL and STL by less than $0.5 \mathrm{~dB}$ even at $500 \mathrm{~Hz}$; the difference is much less at lower frequencies. Therefore, use of uncoupled transfer matrices for evaluation of LTL and STL would suffice.

FTL values computed with the classical beam assumptions (neglecting shear deformation, rotary inertia, and fluid inertia) turn out to be substantially lower (by $4-5 \mathrm{~dB}$ ) at higher frequencies $(\sim 500 \mathrm{~Hz})$. Therefore, the exact theory (that is, without the classical beam assumptions) has been used here for the parametric studies. These studies for gaseous medium (refrigerant vapor) indicate that vibration isolation [represented by flexural transmission loss (FTL)] would improve with:

(i) Softer hoses (lower storage modulus, $E_{r}$ ) as indicated by Fig. 5 ,

(ii) lossier hoses (higher loss factor, $\eta$ ) as is obvious from Fig. 6,

(iii) rubber hoses instead of aluminum as is clear from Fig. 7 ,

(iv) longer hoses as is borne out by Fig. 8,

(v) more (smaller) hoses in series with metallic (steel) joints as indicated by Fig. 9,

(vi) thinner walls as is obvious from Fig. 10, and

(vii) lower internal radius as is borne out by Fig. 11 .

$$
\left[T_{f p}\right]=\left[\begin{array}{cc}
\sigma C_{2}-C_{0} & \frac{\nu b}{h}\left[C_{1}-(\sigma+\tau+\gamma) C_{3}\right] \\
2 \nu \sigma \tau C_{3} & (\tau+\gamma) C_{2}-C_{0} \\
2 \nu \sigma C_{2} & \frac{1}{\tau}\left((\tau+\gamma) C_{1}-\left[(\tau+\gamma]^{2}+\sigma \gamma\right] C_{3}\right) \\
\sigma\left(C_{1}-\sigma C_{3}\right) & -\frac{\nu b}{h} \sigma C_{2}
\end{array}\right.
$$

In particular, Fig. 9 shows that the effect of a multiple hose is similar to that of a multiple expansion chamber muffler: better at higher frequencies, but worse at lower frequencies.

Bends have a mixed but generally beneficial effect on vibration isolation (see Fig. 12), and therefore may be used as necessitated by logistics.

All these observations, viz., (i)-(vii), are in fact independent of the fluid medium inside the hose pipe, inasmuch as they have been found to hold for liquid medium well.

Finally, it may be noted that this paper has been concerned primarily with vibration isolation of hoses. For applications dealing primarily with sound waves along hoses or hose mufflers like power-steering systems, ${ }^{3,4,8,9}$ the theory developed in Ref. 5 with that of Ref. 6 as the base would be more useful inasmuch as it would help in evaluating the breakout noise or transverse transmission loss (TLtp) that imposes a limit on effectiveness of axial sound TL (STL) realization.

\section{ACKNOWLEDGMENTS}

The work reported here was done at Ford Motor Company when the first author was on sabbatical leave from Indian Institute of Science, Bangalore. The authors thankfully acknowledge the support and permission to publish by the Climate Control Operations of the Automotive Components Division of Ford Motor Company.

\section{APPENDIX A: TRANSFER MATRICES}

The field transfer matrix of a beam element is composed of submatrices representing different types of waves. For Poisson coupled axial stress waves in the hose wall and pressure waves in the fluid inside, the nondimensional representation of the field transfer matrix and state vector are ${ }^{2}$

$$
\left.\begin{array}{cc}
\frac{\nu b}{h} \tau C_{2} & -C_{1}+(\sigma+\gamma) C_{3} \\
\tau\left[(\tau+\gamma) C_{3}-C_{1}\right] & -2 \nu \tau C_{2} \\
(\tau+\gamma) C_{2}-C_{0} & 2 \nu\left[(\sigma+\tau+\gamma) C_{3}-C_{1}\right] \\
-\frac{\nu b}{h} \sigma \tau C_{3} & \sigma C_{2}-C_{0}
\end{array}\right],
$$

where subscript $f p$ indicates sound waves in the fluid and longitudinal waves along the pipe wall,

$$
\begin{gathered}
\tau=\frac{\omega^{2} l^{2}}{a_{f}^{2}}, \\
\sigma=\frac{\omega^{2} l^{2}}{a_{p}^{2}}, \\
\gamma=2 \nu^{2} \frac{b \sigma}{d},
\end{gathered}
$$

$$
\begin{aligned}
& b=\frac{\tau}{e}, \\
& d=\frac{\rho_{p}}{\rho_{f}}, \\
& h=\frac{E}{K^{*}}, \\
& C_{0}=\Delta\left[\lambda_{2}^{2} \cos \left(\lambda_{1}\right)-\lambda_{1}^{2} \cos \left(\lambda_{2}\right)\right],
\end{aligned}
$$




$$
\begin{aligned}
& C_{1}=\Delta\left[\frac{\lambda_{2}^{2}}{\lambda_{1}} \sin \left(\lambda_{1}\right)-\frac{\lambda_{1}^{2}}{\lambda_{2}} \sin \left(\lambda_{2}\right)\right], \\
& C_{2}=\Delta\left[\cos \left(\lambda_{1}\right)-\cos \left(\lambda_{2}\right)\right], \\
& C_{3}=\Delta\left[\frac{1}{\lambda_{1}} \sin \left(\lambda_{1}\right)-\frac{1}{\lambda_{2}} \sin \left(\lambda_{2}\right)\right], \\
& \Delta=\left[\lambda_{1}^{2}-\lambda_{2}^{2}\right]^{-1}, \\
& \lambda_{1}^{2}=\frac{1}{2}\left((\tau+\sigma+\gamma)-\left[(\tau+\sigma+\gamma)^{2}-4 \sigma \tau\right]^{1 / 2}\right),
\end{aligned}
$$

$$
\lambda_{2}^{2}=\frac{1}{2}\left((t+\sigma+\gamma)+\left[(\tau+\sigma+\gamma)^{2}-4 \sigma \tau\right]^{1 / 2}\right),
$$

and the nondimensional state vector at location $i$ (the righthand end) in Fig. 1 is

$$
Z_{i}=\left(\frac{U_{z}}{l} \frac{p}{K^{*}} \frac{V}{l} \frac{F_{z}}{A_{p} E}\right)_{i}^{T} .
$$

For flexural vibration of the hose in the $y-z$ plane, the nondimensional representation of the field transfer matrix and state vector are ${ }^{2}$

$$
\left.\begin{array}{c}
-\frac{1}{\gamma}\left[-\sigma C_{1}+\left(\gamma+\sigma^{2}\right) C_{3}\right] \\
C_{2} \\
{\left[C_{1}-(\sigma+\tau) C_{3}\right]} \\
C_{0}-\sigma C_{2}
\end{array}\right],
$$

$$
\begin{aligned}
& \sigma=\frac{\left(\rho_{p} A_{p}+\rho_{f} A_{f}\right)}{G A_{p} k_{s}} \omega^{2} l^{2}, \\
& \tau=\frac{\left(\rho_{p} I_{p}+\rho_{f} I_{f}\right)}{E I_{p}} \omega^{2} l^{2}, \\
& \gamma=\frac{\left(\rho_{p} A_{p}+\rho_{f} A_{f}\right)}{E I_{p}} \omega^{2} l^{4}, \\
& C_{0}=\Delta\left[\lambda_{4}^{2} \cosh \left(\lambda_{3}\right)+\lambda_{3}^{2} \cos \left(\lambda_{4}\right)\right], \\
& C_{1}=\Delta\left[\frac{\lambda_{4}^{2}}{\lambda_{3}} \sinh \left(\lambda_{3}\right)+\frac{\lambda_{3}^{2}}{\lambda_{4}} \sin \left(\lambda_{4}\right)\right], \\
& C_{2}=\Delta\left[\cosh \left(\lambda_{3}\right)-\cos \left(\lambda_{4}\right)\right], \\
& C_{3}=\Delta\left[\frac{1}{\lambda_{3}} \sinh \left(\lambda_{3}\right)-\frac{1}{\lambda_{4}} \sin \left(\lambda_{4}\right)\right], \\
& \Delta=\left[\lambda_{3}^{2}+\lambda_{4}^{2}\right]^{-1}, \\
& \lambda_{3}^{2}=\left(\gamma+\frac{1}{4}(\sigma-\tau)^{2}\right)^{1 / 2}-\frac{1}{2}(\sigma+\tau), \\
& \lambda_{4}^{2}=\left(\gamma+\frac{1}{4}(\sigma-\tau)^{2}\right)^{1 / 2}+\frac{1}{2}(\sigma+\tau),
\end{aligned}
$$

The state vector in the $y-z$ plane at location $i$ (right-hand end) in Fig. 1 is

$$
Z_{i}=\left(\frac{U_{y}}{l} \psi_{x} \frac{M_{x} l}{E I_{x}} \frac{F_{y} l^{2}}{E I_{p}}\right)_{i}^{T} .
$$

\section{General field transfer matrix}

The field transfer matrix for a single straight pipe reach shown in Fig. 1 is composed of two submatrices: longitudinal vibration of the liquid and pipe wall and transverse vibration in the $y-z$ plane (neglecting torsion). Their expressions were given in Eqs. (A1) and (A3), respectively. The state vectors have eight dependent variables: two for each of the forces, moments, displacements, and rotations of the pipe wall, and one each for acoustic pressure and particle displacement of the fluid inside. The equation below shows these arrangements:

$$
Z_{i}=\left[T_{L}\right] Z_{i-1},
$$

where $\left[T_{L}\right]$ is the field transfer matrix for a pipe reach of length $l$ in the local coordinate system. This $8 \times 8$ matrix may be partitioned as ${ }^{2}$

$$
\left[T_{L}\right]=\left[\begin{array}{cc}
{\left[T_{f p}\right]} & 0 \\
0 & {\left[T_{y z}\right]}
\end{array}\right] .
$$

The state vector at location $i$ in Fig. 1 is

$$
Z_{i}=\left(\frac{U_{z}}{l} \frac{P}{K^{*}} \frac{V}{l} \frac{F_{z}}{A_{p} E} \frac{U_{y}}{l} \Psi_{x} \frac{M_{x} l}{E I_{p}} \frac{F_{y} l^{2}}{E I_{p}}\right)^{T} .
$$

\section{Transfer matrix for a bend}

The point transfer matrix relation for a sharp bend (see Fig. 2) is ${ }^{2}$ 


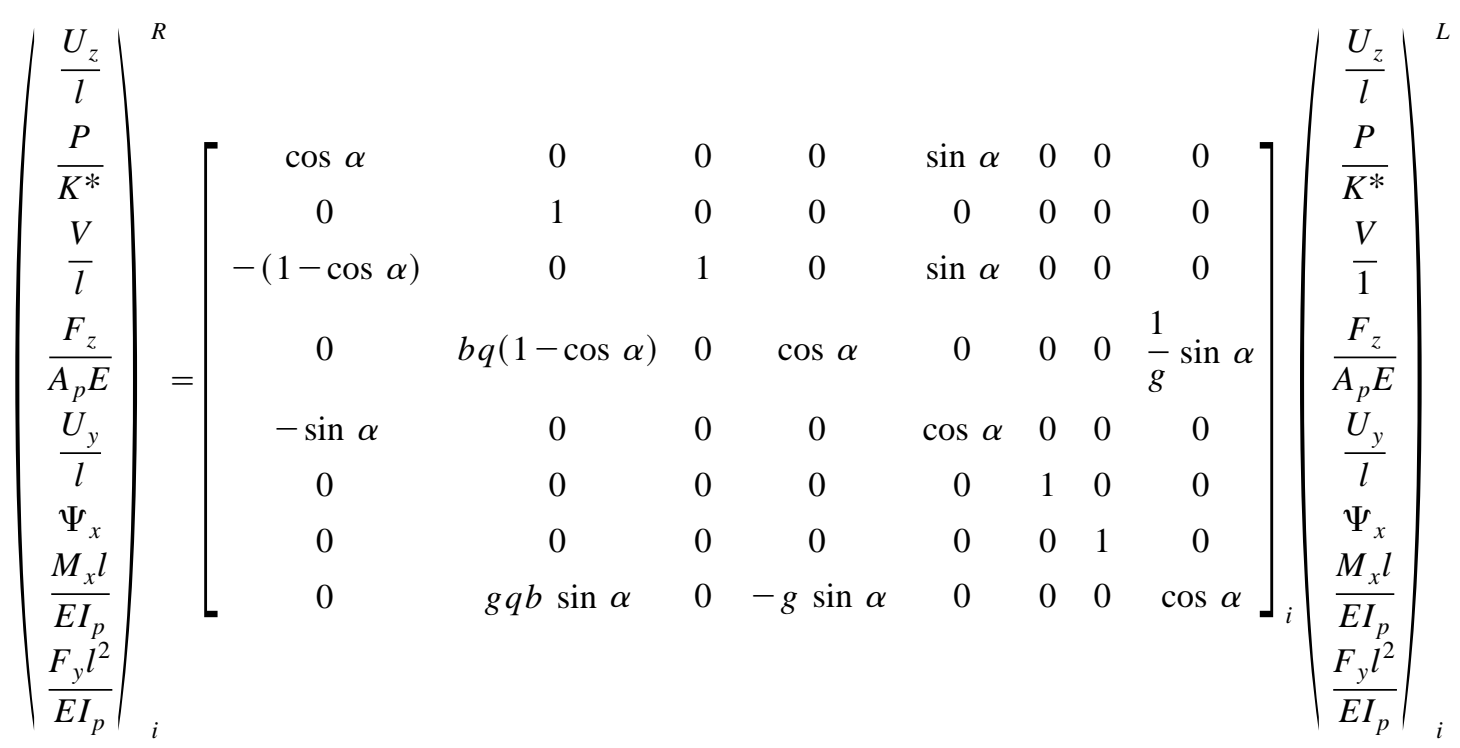

or

$$
Z_{i}^{R}=\left[P_{L}^{B}\right]_{i} Z_{i}^{L},
$$

where superscripts $R, B$, and $L$ denote right, bend, and left respectively, and

$$
\begin{gathered}
g=\frac{A_{p} l^{2}}{I_{p}}, \\
q=\frac{A_{f}}{A_{p}}, \\
b=\frac{K^{*}}{E} .
\end{gathered}
$$

\section{APPENDIX B: HOSE DIMENSIONS AND MATERIAL PROPERTIES USED IN THE PARAMETRIC STUDIES}

Inside fluid: default: HFC-134a refrigerant vapor density $=22 \mathrm{~kg} / \mathrm{m}^{3}$, bulk modulus $=4.436 \times 10^{5} \mathrm{~Pa}$

alternative: power-steering system oil density $=834 \mathrm{~kg} / \mathrm{m}^{3}$, bulk modulus $=1.5 \times 10^{9} \mathrm{~Pa}$

Hose material: default: $3 / 4$ in. hose used in climate control system

Elastic modulus $E=E_{r}(1+j \eta)$

Storage modulus,

$$
E_{r}=2.0 \times 10^{8}(1+f / 1000), \mathrm{Pa}
$$

Loss factor, $\eta=0.2(1+0.1 f / 1000)$

Poisson's ratio, $\nu=0.48$

Internal radius, $r=9.5 \mathrm{~mm}$

Wall thickness, $e=4 \mathrm{~mm}$

Density, $\rho=1196 \mathrm{~kg} / \mathrm{m}^{3}$

alternative: aluminum hose

Storage modulus, $E_{r}=6.9 \times 10^{10} \mathrm{~Pa}$

Loss factor, $\eta=0.0001$
Poisson's ratio, $\nu=0.33$

Density, $\rho=2710 \mathrm{~kg} / \mathrm{m}^{3}$

Internal radius, $r=8 \mathrm{~mm}$

Wall thickness, $e=2 \mathrm{~mm}$

Terminal material: mild steel

Storage modulus, $E_{r}=2.1 \times 10^{11} \mathrm{~Pa}$

Loss factor, $\eta=0.0001$

Poisson's ratio, $n=0.29$

Density, $\rho=7800 \mathrm{~kg} / \mathrm{m}^{3}$

Internal radius, $r=$ hose radius

Wall thickness, $e=$ hose wall thickness

Flexural transmission loss (FTL) has been selected for parametric studies on vibration isolation.

${ }^{1}$ D. C. Wiggert, R. S. Otwell, and F. J. Hatfield, "The effect of elbow restraint on pressure transients," ASME J. Fluids Eng. 107, 402-406 (1995).

${ }^{2}$ M. W. Lesmez, D. C. Wiggert, and F. J. Hatfield, "Modal analysis of vibrations in liquid-filled piping systems," ASME J. Fluids Eng. 112, 311-318 (1990).

${ }^{3}$ L. Suo and E. B. Wylie, "Complex wave speed and hydraulic transients in viscoelastic pipes,'” ASME J. Fluids Eng. 112, 496-500 (1990).

${ }^{4}$ M. C. Hastings and Chuan-Chiang Chen, "Analysis of tuning cables for reduction of fluid borne noise in automotive power steering hydraulic line," SAE paper No. 931295, Noise and Vibration Conference in Traverse City, 1994.

${ }^{5}$ M. L. Munjal and P. T. Thawani, "A simple model for wave propagation along and across a hose," Proceedings of Inter-Noise 95 (1995), pp. 361366.

${ }^{6}$ M. L. Munjal, Acoustics of Ducts and Mufflers (Wiley-Interscience, New York, 1987)

${ }^{7}$ L. Cremer, M. Heckl, and E. E. Ungar, Structure-borne Sound (SpringerVerlag, Berlin, 1988), 2nd ed.

${ }^{8}$ D. K. Longmore and A. Schlesinger, "Transmission of Vibration and pressure fluctuations through hydraulic hoses," Proc. Inst. Mech. Eng. 205, 97-104 (1991).

${ }^{9}$ Jean Botti, G. Venizelos, and N. Benkaza, "Optimization of power steering systems vibration reduction in passenger cars," in SAE Proceedings of the 1995 Noise and Vibration Conference, pp. 1179-1201, paper No. 951253. 